\title{
Thrombosis Heralding Aneurysmal Rupture: An Exploration of Potential Mechanisms in a Novel Giant Swine Aneurysm Model
}

\author{
J. Raymond, T.E. Darsaut, M. Kotowski, A. Makoyeva, G. Gevry, F. Berthelet, and I. Salazkin
}

\begin{abstract}
BACKGROUND AND PURPOSE: The relationship between aneurysm dimensions, flow, thrombosis, and rupture remains poorly understood. We attempted to clarify this relationship by exploring various swine aneurysm models.

MATERIALS AND METHODS: Bilateral carotid aneurysms were constructed according to 3 protocols in 24 animals: small aneurysms with wide necks (group 1; $n=6$ animals); small aneurysms with small necks (group 2; $n=4$ animals), and giant aneurysms with large necks (group 3; $n=14$ animals). Group 3 included 3 subgroups, related to testing the model in various experimental conditions: The neck was clipped in 3 animals; venous pouches lacked an endothelial lining in 4 animals; and 7 were control animals. Animals were followed until rupture, or for 1-4 weeks. Angiography was performed postoperatively and before euthanasia. We studied lesion pathology, paying attention to thrombosis, recanalization, wall composition, and perianeurysmal hemorrhage.

RESULTS: Groups differed significantly in aneurysm dimensions and aspect ratio $(P=.002)$. Ruptures occurred more frequently in animals with untreated giant aneurysms $(7 / 7)$ than in animals with small wide-neck $(0 / 6)$ or small-neck $(2 / 4)$ aneurysms $(P=.002)$. Ruptures occurred only in animals with thrombosed aneurysms. Lesions lacking an endothelial lining and 5 of 6 clipped venous pouches thrombosed but did not rupture. One giant lesion ruptured despite complete clipping. The wall was deficient in $\alpha$-actin and was infiltrated with inflammatory cells and erythrocytes in all thrombosed cases, ruptured or not. Ruptures were associated with recanalizing channels in 9 of 10 cases.
\end{abstract}

CONCLUSIONS: Thrombosis, inflammation, and recanalization may precipitate aneurysmal ruptures in a swine model

ABBREVIATIONS: $A R=$ aspect ratio; HPS = hematoxylin phloxine saffron

Elow diverters are endovascular stentlike devices with the caFacity for curative reconstruction of large and giant intracranial aneurysms. ${ }^{1}$ Hemorrhagic complications occurring after flow diversion of unruptured aneurysms have been reported, but the incidence of this dreadful complication remains indeterminate. An urgent field notice was made public in March 2010, reporting 8 deaths from hemorrhages 5-150 days after flow diversion. $^{2}$ The mechanism of post-flow-diversion rupture remains elusive; aneurysm thrombosis, followed by mural inflamma-

Received December 14, 2011; accepted after revision May 16, 2012.

From the Department of Radiology (J.R.), Interventional Neuroradiology Research Laboratory (T.E.D., M.K., A.M., G.G., I.S.), and Department of Pathology (F.B.), Centre Hospitalier de l'Université de Montréal, Notre-Dame Hospital, Montreal, Quebec, Canada; and Division of Neurosurgery (T.E.D.), Department of Surgery, University of Alberta Hospital, Mackenzie Health Sciences Centre, Edmonton, Alberta, Canada.

Please address correspondence to Jean Raymond, MD, CHUM-Notre-Dame Hospital, Interventional Neuroradiology, 1560 Sherbrooke East, Pavilion Simard, Room Z12909, Montreal, Quebec, Canada H2L 4M1; e-mail: jean.raymond@umontreal.ca

Evidence-Based Medicine Level 2.

http://dx.doi.org/10.3174/ajnr.A3407 tion, leading to autolysis and rupture, ${ }^{3}$ or "destabilization" of the aneurysm wall ${ }^{4}$ has been described. Ruptures are also known to have occurred following treatment of giant aneurysms by parent vessel occlusion. ${ }^{5,6}$ Some investigators have proposed that thrombosis may herald ruptures in untreated aneurysms. ${ }^{7,8}$ Thrombusprecipitating rupture has also been implicated in abdominal aortic aneurysms. ${ }^{9-12}$ However, because thrombosis followed by organization and neointimal closure of the neck is required for successful occlusion of aneurysms by endovascular embolization, ${ }^{11,13-18}$ there must be additional factors to account for thrombosis leading to rupture rather than occlusion. Such discriminating factors have not been explored experimentally, to our knowledge.

Some porcine venous pouch carotid aneurysms have a propensity for early rupture, within 3-5 days of surgical construction, when incompletely occluded with coils. ${ }^{19}$ In this work, we explored different variants of a porcine model to systematically study the potential contribution of anatomic factors, including fundus and neck size, residual blood flow, thrombosis, and recanalization, to the events leading to aneurysm wall breakdown. We 


\begin{tabular}{crcccc}
\multicolumn{6}{l}{ Characteristics of the various groups of aneurysms } \\
\multicolumn{7}{c}{${ }^{\mathbf{a}}$} \\
& No. & $\begin{array}{c}\text { Long Axis } \\
(\mathbf{m m})\end{array}$ & $\begin{array}{c}\text { Short Axis } \\
(\mathbf{m m})\end{array}$ & $\begin{array}{c}\text { Neck } \\
(\mathbf{m m})\end{array}$ & Aspect Ratio \\
\hline $\begin{array}{c}\text { Group } \\
1\end{array}$ & 12 & $11.3 \pm 2.6$ & $6.7 \pm 2.1$ & $5.8 \pm 0.6$ & $2.1 \pm 0.6$ \\
2 & 8 & $16.9 \pm 4.0$ & $8.1 \pm 1.3$ & $4.8 \pm 1.1$ & $3.6 \pm 1.0$ \\
3 & 28 & $26.1 \pm 10.9$ & $9.4 \pm 1.4$ & $5.8 \pm 1.0$ & $4.6 \pm 1.8$ \\
$P$ value & & .000 & .01 & .84 & .001 \\
\hline
\end{tabular}

artery) anastomosis, while "giant aneurysms" were made by using a longer venous segment and a side-to-side anastomosis. In a first group of 6 animals, 12 small wideneck (5-7 mm) aneurysms were constructed (Table). Animals were followed for 2,3 , and 4 weeks ( $n=2$ each), but no rupture occurred. In a second group of 4 animals, 8 aneurysms were constructed in a similar fashion, but the arteriotomy was

then tested the final model in 2 experimental conditions to eradicate blood flow by clipping and to produce venous pouches that lacked an endothelial lining. The present work suggests that thrombosis, more frequent in large aneurysms with a high fundus-neck or aspect ratio, can lead to mural degradation, but additional conditions are required for rupture to occur: namely residual blood flow or recanalization. Preserved blood flow seems necessary for blood to dissect between the thrombus and the aneurysm wall, infiltrate the degenerated wall, and rupture into perianeurysmal tissues. This model may prove useful to test strategies to decrease posttreatment hemorrhagic complications or even medical treatments for unruptured aneurysms. ${ }^{20-22}$

\section{MATERIALS AND METHODS \\ Surgical Aneurysm Creation}

Protocols for animal experimentation were approved by the Institutional Animal Care Committee in accordance with guidelines of the Canadian Council on Animal Care. Multiple protocol revisions and committee consultations were necessary to arrive at the final model. All procedures were performed in 20- to 25-kg Yorkshire pigs under general anesthesia. Through a midline vertical cervical incision, the left external jugular vein was harvested, turned inside-out to remove potential valvular obstructions, then reverted, and placed in heparinized saline. The pretracheal fascia was divided to obtain access to both carotid arteries. The left common carotid artery was mobilized, temporary clips were applied, and a 5- to 7-mm arteriotomy was performed on the anterolateral wall. A 10 - to $12-\mathrm{mm}$ venous segment was then sutured end-toside to the arteriotomy, by using a continuous 7.0 Prolene suture (Ethicon, Cincinnati, Ohio). The end of the venous segment was closed with a permanent clip to form a cylindric aneurysm fundus, the height of which varied. The same procedure was repeated on the right common carotid artery to create symmetric bilateral aneurysms.

\section{Aneurysm Subgroups}

A variety of types of aneurysms were created during an exploratory phase, reported in the present article, to find the model that most consistently led to predictable aneurysmal ruptures, while attempting to minimize animal distress and the number of experiments. This variety led to disparities in the number of animals and the length of the follow-up periods between groups described here. The rationale was to explore surgical ways to produce aneurysms with widely different aspect ratios, presumably with different propensities to thrombose and rupture, by varying either the size of the sac or the size of the neck of the aneurysms. "Small aneurysms" were constructed with an end-to-side (vein-to- smaller, between 4 and $6 \mathrm{~mm}$ (group 2, small aneurysms with small necks). This group was followed for 2 weeks or until rupture occurred. In a third group of 14 animals, we adopted the final model: giant $(25-50 \mathrm{~mm})$ aneurysms were created with a much longer venous segment, a side-to-side anastomosis, and a 6- to 7-mm arteriotomy (group 3, giant aneurysms with wide necks; $n=14$; Fig 1).

\section{Other Experimental Conditions}

To explore mechanisms involved in ruptures and to provide pathologic specimens of thrombosed venous pouches that would not rupture, we submitted the final model to 2 other conditions: 1) To study effects of blood flow on the incidence of ruptures and on the pathologic findings, we permanently clipped the neck after surgery in 3 animals (6 lesions); and 2) to promote thrombosis and prevent early recanalization of the clot, we constructed giant pouches lacking an endothelial lining by leaving the vein segment inside-out, as previously described ( $n=4$ animals; 8 lesions; Fig 2). ${ }^{23,24}$

\section{Angiography, Euthanasia, and Pathology}

Selective conventional angiography was performed immediately following surgery with $5 \mathrm{~F}$ catheters via a percutaneous femoral approach. Aneurysm dimensions were measured by using the catheter caliber to correct for magnification. Pigs recovered from surgery and were fed a normal diet. Postoperatively, animals were observed for possible pain, distress, or airway compromise from a neck hematoma. For pigs that did not experience fatal hemorrhage, angiography was performed before euthanasia.

Autopsy was performed within 1 day of a fatal hemorrhage $(n=5)$ or after elective euthanasia by barbiturate overdose at 7 days for group 3 animals $(n=11)$ or later for group 1 or 2 preliminary animals at $2(n=4), 3(n=2)$, or 4 weeks $(n=2)$. Large cervical hematomas, when present, were explored to identify the side and site of aneurysm rupture. Carotid aneurysm constructs were removed en bloc and immersed in formalin for 1 week. Lesions were measured and photographed; carotid arteries were first opened longitudinally; then, aneurysms or venous pouches were opened along the midsagittal plane. Multiple microscopic photographs of the content and wall were taken by using a computerized image analysis system (Vision 2.0; Clemex Technologies, Longueuil, Canada). All specimens were then sectioned; embedded in paraffin; and stained with HPS, Movat pentachrome, and immunohistochemical markers for smooth-muscle cell $\alpha$ actin and Factor VIII. Pathology slides were examined by 2 experienced observers (I.S. and J.R.), aware of treatment allocation, 

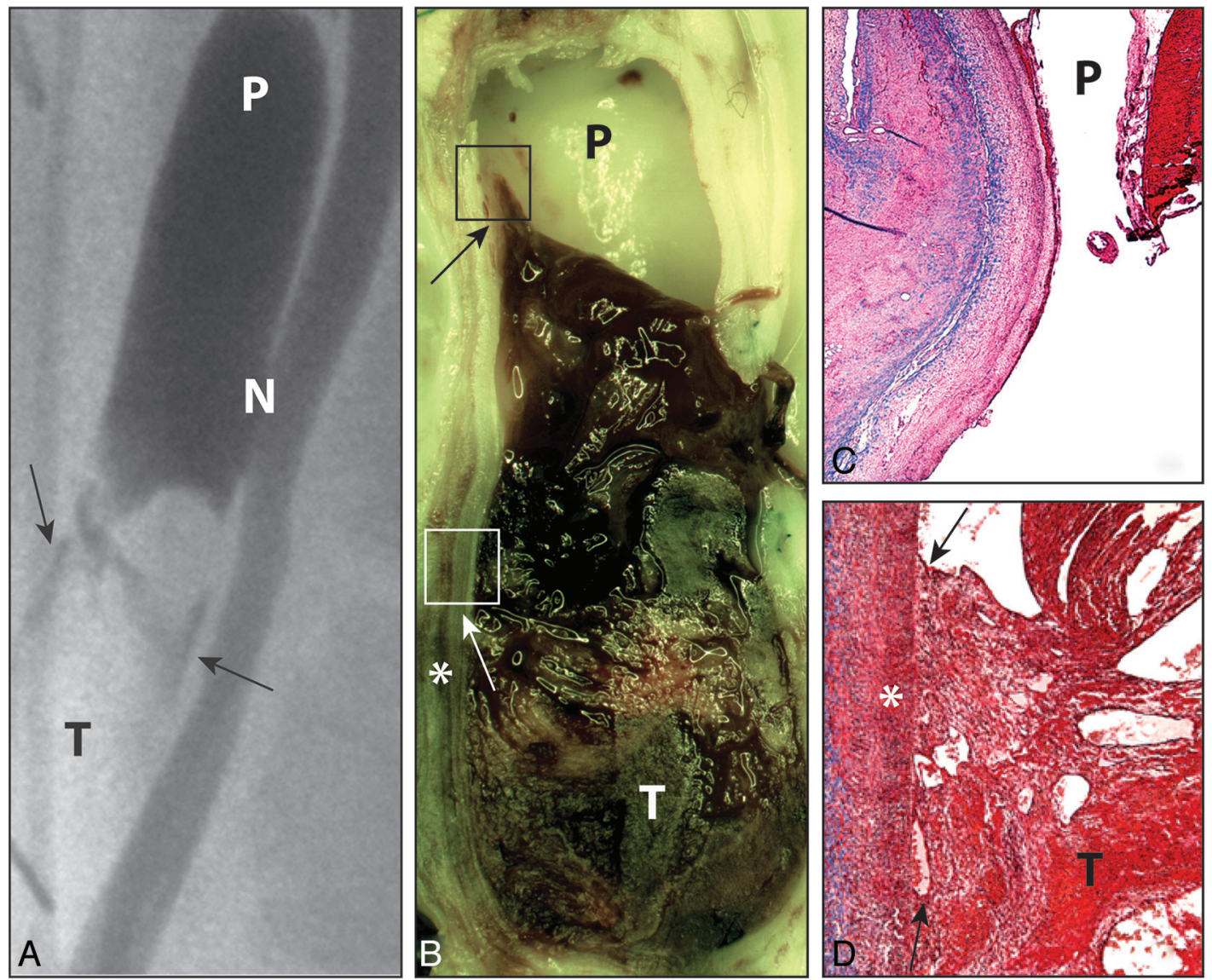

FIG 1. Untreated ruptured giant aneurysm. Angiography $(A)$, sagittal sections $(B)$, and microscopic sections $(C$ and $D)$ of a control giant aneurysm 7 days after construction and rupture. Note the thrombosed caudal third (T), the flowing cephalad third (P), and the partially thrombosed middle third of the fundus. While there is neointimal hypertrophy of the wall of the cephalad third $(C)$, there is thrombosis with recanalization of the caudal section and hemorrhagic transformation of the wall (asterisk, $B$ and $D$ ). To demonstrate patency of the aneurysm, one must catheterize the aneurysm and inject contrast directly into its lumen. Note recanalization between the clot and wall (arrows in $A$ and $D$ ). Movat pentachrome, original magnification $\times 20$. $\mathrm{N}$ indicates aneurysm neck.

correlating presacrifice angiography with photography and gross pathologic findings (Figs 1-3).

\section{Statistics}

Continuous variables were compared by using ANOVA, while categoric variables were analyzed by using $\chi^{2}$ tests for multiple comparisons, with a $P$ value of .05 as significant. Rupture rates were compared by using animals, not aneurysm numbers.

\section{RESULTS}

\section{Group Characteristics}

Aneurysm characteristics of the various groups are summarized in the Table. The mean long-axis aneurysm dimension $(P=.000)$ and mean aspect ratio $(P=.002)$ among the 3 groups were significantly different. Mean neck size was not significantly different among the 3 groups $(P=.84)$.

\section{Aneurysm Ruptures}

Ruptures were significantly more frequent in animals with untreated giant wide-neck aneurysms (group 3 ) than in the 2 other groups $(P=.002)$. Aneurysm ruptures occurred in $0 / 6$ of the group 1 animals (small aneurysms with wide necks) within 4 weeks of follow-up. Two of 4 group 2 animals (small aneurysms with small necks) had fatal ruptures within 2 weeks of follow-up. All aneurysms of untreated group 3 animals (giant aneurysms with wide necks) ruptured, 3/7 fatally and 4/7 with large but nonlethal cervical hematomas, which were documented postmortem on day 7 .

None of the giant aneurysms lacking an endothelial lining ruptured ( 4 animals) $(P=.002$ compared with untreated giant aneurysms), while 1 clipped lesion ruptured (1 of 6 in 3 animals), causing a nonlethal cervical hematoma that appeared on day 5 and increased in size on day 7 , before angiography and euthanasia.

All fatal ruptures occurred on day $4(n=5)$; it was difficult to determine the exact timing of the nonlethal hematomas $(n=5)$. All hemorrhages were identified by day 7 , but in 2 animals, multiple sublethal ruptures were suspected before sacrifice on day 7. It was often difficult to identify the side of the rupture, with hematoma transgressing tissue planes disrupted by surgery.

\section{Angiographic Results}

All group 1,2, and untreated group 3 aneurysms were patent after surgery, but clipped aneurysms (6/6) and de-endothelialized venous pouches $(7 / 8)$ were shown to be occluded on the angiograms obtained immediately after aneurysm creation. Clipped aneurysms were never shown to be patent by angiography. Blood flow 

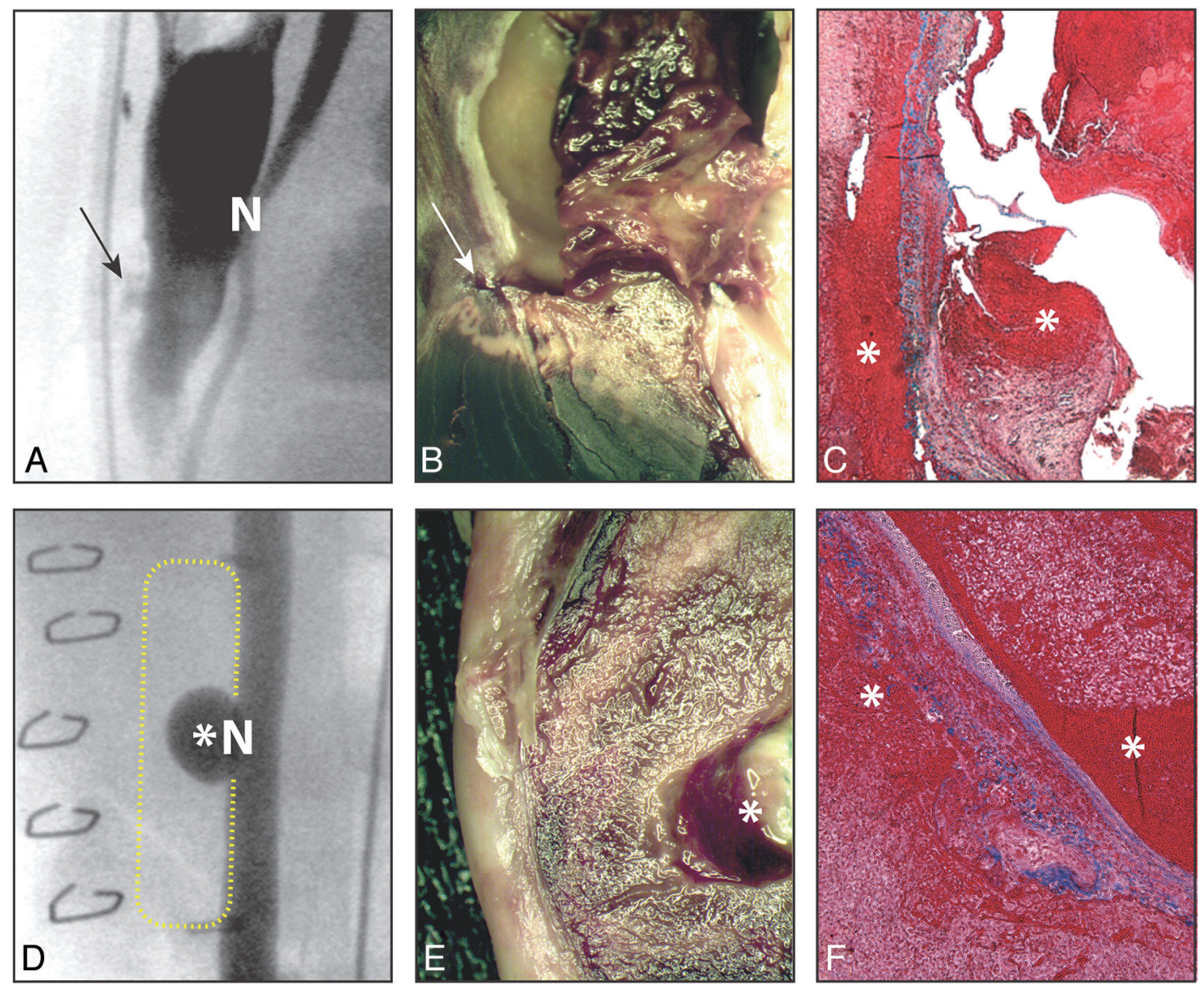

FIG 2. Ruptured and unruptured group 3 aneurysms. Angiography ( $A$ and $D$ ), sagittal section ( $B$ and $E$ ), and microscopic sections ( $C$ and $F) 7$ days after surgery. The arrows in $A$ and $B$ show the rupture site in a control partially thrombosed aneurysm ( $N$ indicates aneurysm neck). The asterisks in $D$ and $E$ show the recurrent aneurysm in a thrombosed lesion lacking an endothelial lining. Note recanalization between the clot and the wall of the control aneurysm, absent in the aneurysm lacking an endothelial lining, and the presence of blood on both sides of the attenuated aneurysm wall media in both cases (asterisks, $C$ and F). $C$ and F, Movat pentachrome, original magnification $\times 20$.

in the giant aneurysms was slow, and selective carotid injections of contrast material barely penetrated the aneurysms; intra-aneurysmal catheterization was necessary to adequately demonstrate patency.

Small wide-neck aneurysms remained patent at 2 weeks, became partially occluded at 3 weeks, and were completely occluded by 4 weeks. Small aneurysms with narrow necks were fully occluded at 2 weeks in the 2 animals whose aneurysms did not rupture.

Giant aneurysms were partially thrombosed in all untreated animals at 7 days (Figs 1 and 2). The caudal portion of the venous pouches was usually more completely thrombosed than the cephalad portion (Fig 1A). At day 7, the site of sublethal rupture was thought to be detected by angiography in 2 cases (Fig $2 A$ ). Sacs lacking an endothelial lining were thrombosed immediately postoperatively (7/8), but a small wide-neck saccular remnant was present by day 7 in all cases (Fig 2D). The 6 lesions that were clipped were confirmed to be completely occluded immediately postoperatively and at 7 days (including the lesion that ruptured). It was not possible to perform antemortem angiography in the animals with fatal aneurysm ruptures.

\section{Pathologic Results}

Group 1: Small Aneurysms with Wide Necks ( $n=6$ animals). None of these aneurysms had ruptured; aneurysms that remained patent at 2 and 3 weeks had substantially thickened hypertrophied walls infiltrated with myofibroblasts and collagen and thick neointima lining the aneurysm lumen. Aneurysms that were occluded at 4 weeks had complete neointimal closure of the neck, with organized thrombus filling the aneurysm lumen (not shown).

Group 2: Small Aneurysms with Small Necks ( $n=4$ animals). Two animals were euthanized at 2 weeks; all 4 aneurysms were completely occluded, with full neointimal closure of the neck. The aneurysm was filled with thrombus in various stages of organization in both cases. The 2 other animals had fatal ruptures on day 4 . In 1 case, the rupture clearly occurred on the side with a nearly completely thrombosed aneurysm, while the contralateral aneurysm remained patent. A recanalizing crescent, lined with endothelial cells, could be followed up to the rupture site (Fig 4). In the other animal, a definite site of hemorrhage could not be identified. Both aneurysms were partially thrombosed and recanalized. Blood had diffusely infiltrated perianeurysmal tissues on both sides.

Group 3: Large or Giant Aneurysms with Wide Necks ( $n=14$ animals) (Control Group). All animals with untreated large and giant aneurysms $(n=7)$ had hemorrhages; 3 proved fatal. It was unclear which aneurysm had ruptured at autopsy in 4 of 7 cases; 

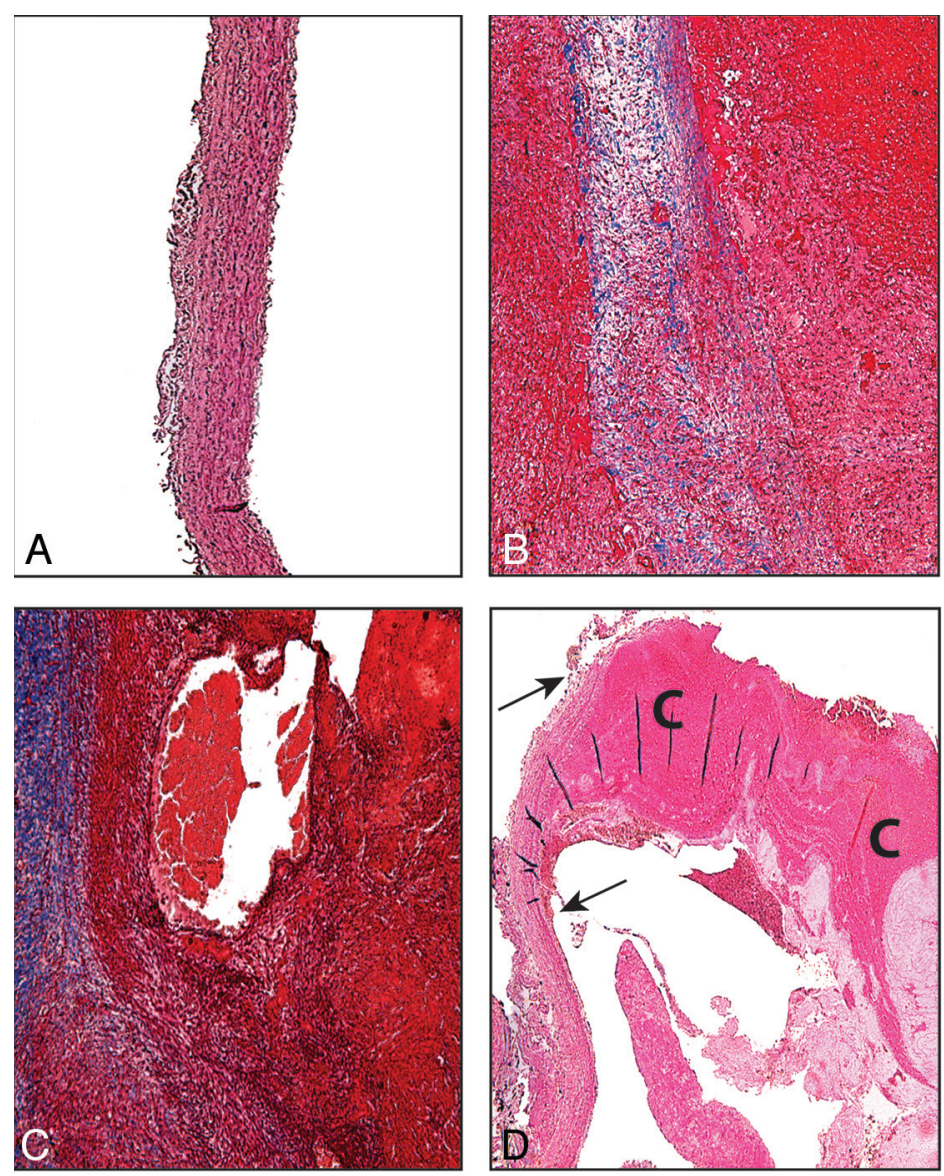

FIG 3. Aneurysm wall. Microscopic section of the wall of the aneurysms, $4(A$ and $D)$ and $7(B$ and $C$ ) days after surgery. Note normal wall $(A)$, thickened, attenuated wall in a thrombosed aneurysm with hemorrhagic infiltration of the adventitia $(B$ and $C)$, with $(C)$ or without $(B)$ recanalization. Note extrusion of endoaneurysmal clot $(C)$ through complete rupture of the wall (arrow, D). HPS ( $A$ and $D)$ and Movat pentachrome $(B$ and $C)$, original magnification $\times 20$ $(A$ and $D), \times 50$ ( $B$ and $C)$.

all soft tissues were infiltrated with blood. In 2 cases, we identified rupture sites on both left- and right-sided aneurysms; in 1 aneurysm, we found 2 distinct aneurysm rupture sites. Ten of 14 aneurysms of this group were completely or nearly completely filled with thrombus. At microscopy, thrombus was adherent to the aneurysm wall; the wall was no longer recognizable as a boundary between intra- and extra-aneurysmal blood (Figs $2 C$ and $3 B$ ). In many areas, smooth-muscle cells and elastic fibers were absent, with a profusion of inflammatory cells infiltrating the wall, along with hemorrhagic transformation of the media, adventitia, and perianeurysmal tissues (Fig 2C). Hemorrhagic wall transformation was always adjacent to the thrombosed portion of the aneurysm. Recanalizing crescents and fissures were clearly seen between the thrombus and the degenerated wall (Fig 3) in 9 of 10 thrombosed aneurysms (Fig 4). They were lined with endothelial cells in 7 cases.

Group 3: Large or Giant Aneurysms with Wide Necks ( $n=14$ animals) (Treated Groups [ $n=7$ animals]). Lesions without endothelium $(n=8)$ or that were clipped $(n=6)$ were completely thrombosed (Fig 2E). Recanalizing crescents were absent ( 6 of 7 cases). Walls remained infiltrated with inflammatory and red blood cells and contained few smooth-muscle cells and elastic fibers. In some places, the wall was nearly absent, as in the control (ruptured) giant aneurysms (Fig 2F). No lumen could be found in the clipped lesion that ruptured. The sac nonetheless had recanalizing channels between the wall and the thrombus (Fig 5). Numerous engorged vasa vasorum were found in the wall and perilesional tissues, but this observation was reproducibly found in all aneurysms studied at 7 days, ruptured or not. In de-endothelialized venous pouches, the thrombus was covered with early neointimal tissue, including endothelial cells, delineating a neoaneurysm of $5-10 \mathrm{~mm}$.

\section{DISCUSSION}

The main findings of this work are the following: 1) Ruptures occur more frequently when the aneurysm is large or giant and the neck size is small relative to the pouch (ie, large aspect ratios); 2) thrombosis, partial or complete, is consistently found in ruptured aneurysms; 3) thrombosis is associated with inflammation and hemorrhagic wall transformation, with partial-to-complete disappearance of elastic fibers and smooth-muscle cells, whether aneurysms rupture or not; 4) ruptures are most often associated with residual blood flow or with evidence suggesting that flow is re-established between the thrombus and the degenerated wall; 5) the endothelial lining of the aneurysm seems to play an important role, because rupture was prevented in 8 of 8 thrombosed giant lesions lacking endothelium; and 6) clipping of giant lesions prevented rupture in most but not all cases (5/6).

\section{Aspect Ratio and Giant Aneurysm Model}

Venous pouch carotid experimental swine aneurysms have frequently been used to evaluate the efficacy of neurovascular devices. ${ }^{11,19,25}$ They are not currently considered appropriate for this purpose because of their propensity for thrombosis and complete closure of aneurysmal necks with thick neointima formation in all animals at 1 month, no matter what material is used. Reports of experimental aneurysms in other species (eg, canines and rabbits) have demonstrated a much greater incidence of long-term patency and a lower incidence of spontaneous hemorrhage (essentially 0 ) than were observed in our study. These may thus be more reliable models for testing of devices. ${ }^{26,27}$ The importance of producing a neck-sac ratio to generate aneurysms that will remain patent was emphasized in German and Black's original publication. ${ }^{28}$ More recently, this ratio has been proposed as a predictive factor for rupture of human aneurysms. ${ }^{7,8}$ Multiple versions of this concept have been described, including an aneurysm volume/neck area ra- 

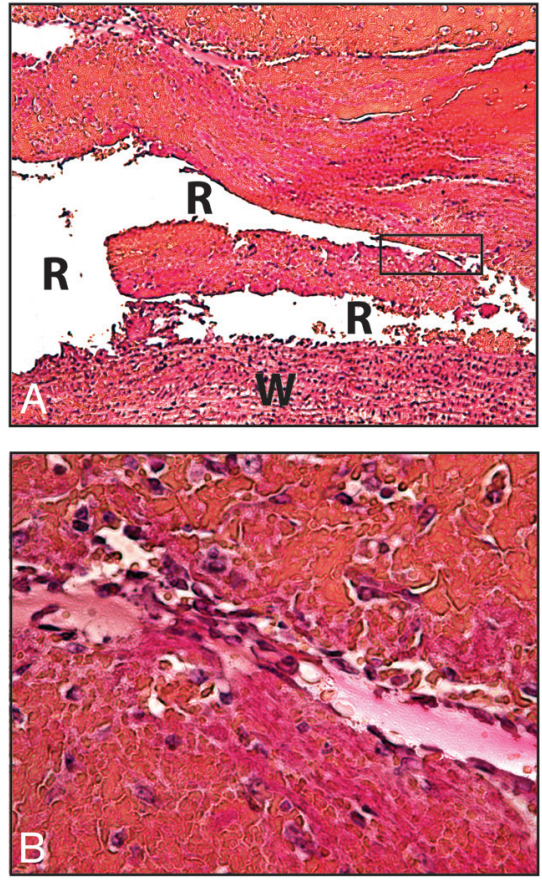

FIG 4. Recanalization and rupture. Recanalized endothelialized crescents can be found in thrombosed aneurysms as early as 4 days after aneurysm construction and rupture. $A, W$ indicates venous wall; $\mathrm{R}$, recanalization. HPS, original magnification $\times 50(A)$. Original magnification $\times 100(B)$.

tio. ${ }^{29}$ Low-flow conditions in experimental aneurysms have been found by using sonography when the aspect ratio is above $1: 6$, a measurement that correlates with ruptured as opposed to unruptured human aneurysms. ${ }^{7,8}$

The relationship between aspect ratio and spontaneous rupture has not been mentioned in the literature in swine models, but our hypothesis is compatible with findings in previous works. According to Byrne et al, ${ }^{19}$ ruptures occurred in 4 of 4 control animals and in 3 of 10 coiled aneurysms when aneurysms were $15 \mathrm{~mm}$ with 4-mm necks (corresponding AR of 3.0-3.75). Murayama et $\mathrm{al}^{30}$ reported constructing 12-mm aneurysms with 7-mm necks (AR, 1.7) with rupture in 3 of 23 (13\%) of the coiled aneurysms. Lee et $\mathrm{al}^{11}$ studied deliberate immediate aneurysm thrombosis and did not mention ruptures in 8-mm aneurysms with 2.5 - $\mathrm{mm}$ necks (AR, 3.2), but "if the aneurysm was considered to be incompletely thrombosed" at operation, "an additional suture or temporary clip was placed on the aneurysm neck for 20 minutes." Furthermore, recanalization occurred in 4 animals that were excluded from further analyses. ${ }^{11}$

The giant aneurysm model described here led to constant, occasionally incomplete thrombosis, with clinical or subclinical ruptures in all cases. This model could find 3 applications: 1) to study hemorrhagic complications after thrombosis (such as following flow diver$\left.\operatorname{sion}^{3}\right) ; 2$ ) to explore mechanisms heralding rupture of unruptured aneurysms; and 3) to test medical treatments for unruptured aneurysms, such as acetylsalicylic acid, anti-inflammatory agents, or matrix metalloproteinase inhibitors. ${ }^{20-22,31,32}$

\section{Hemorrhagic Complications of Flow Diversion}

Other than thrombus, the 4 features common to aneurysms that ruptured following flow diversion, as described by Kulscár et al, ${ }^{3}$ are the following: 1) large or giant size, 2) symptomatic lesions, 3) saccular morphology with $\mathrm{AR}>1.6$ (mean AR, $3.1 \pm 0.9$ ), and 4) inertia-driven aneurysmal inflow. Because thrombus eventually occurs in cases of successful endovascular treatment without ruptures, the presence of thrombus alone cannot be a discriminating factor. Alternative explanations, such as failure to produce complete stasis, perhaps combined with redirection of flow to a weaker portion of the aneurysm wall or with increased aneurysmal pressures, could be evoked as possible mechanisms. While some have used pretreatment computational fluid dynamics studies to analyze potential causes of posttreatment ruptures, ${ }^{33-35}$ hoping to predict future complications via "virtual" elevation of intra-aneurysmal pressure, this hypothesis remains controversial. ${ }^{36}$

This novel swine aneurysm model reproduced 2 possibly essential characteristics of ruptured aneurysms observed in clinical series: large size and aspect ratio. None of these animals were treated with flow diverters, and none had inertia-driven inflows.

\section{Thrombosis Followed by Ruptures}

If stasis caused by a flow diverter can cause thrombosis followed by either favorable evolution or rupture, what discriminates these divergent outcomes? Our findings suggest that thrombosis is accompanied by degeneration of the aneurysm wall, as found in other experimental or human aortic aneurysms. ${ }^{9-12}$ Inflammation of the venous wall was present in unruptured and in ruptured cases. In our model, the distinguishing factor was the presence of recanalization between the thrombus and the necrosing wall. Recanalization and recirculation into an aneurysm with a degenerated wall seem to cause rupture in this model. We then produced thrombosed venous pouches that would presumably lead to a degenerated wall but that would not rupture. Recanalization was prevented by surgical clipping ( $n=5$ of 6 lesions) or by creating venous pouches without an endothelium $(n=8)$. A vasa vasorum theory has been advanced to explain how ruptures can still occur in completely occluded aneurysms. ${ }^{37}$ It would be imprudent to draw conclusions from the single case we encountered.

Endothelial denudation can prevent recanalization after coil embolization of arteries and aneurysms. ${ }^{23}$ A similar recanalization mechanism could explain how restoring flow between the clot and the degenerated wall could precipitate rupture despite partial thrombosis (Figs 3 and 4). The presence of small recurring wide-neck aneurysms in all cases of denuded giant aneurysms shows that the absence of ruptures was not caused by technical failure of aneurysm construction. Recanalization and blood flow between the thrombus and the degenerated aneurysm wall, present in the untreated giant aneurysms that ruptured but absent in denuded giant aneurysms that thrombosed but did not rupture, may be the key features indicating why thrombosis may sometimes lead to rupture and sometimes to healing of the aneurysm. It is unclear whether coiling the aneurysm in addition to flow diversion can prevent hemorrhages, ${ }^{3}$ but complete coiling, as opposed to incomplete coiling, can prevent ruptures of porcine aneurysms. ${ }^{19}$ We chose to clip rather than coil to occlude venous pouches in the present work for many reasons: 1) An enormous amount of coils would have been necessary to completely occlude many giant aneurysms; 2) resulting coil masses would have made detailed pathologic examination of 

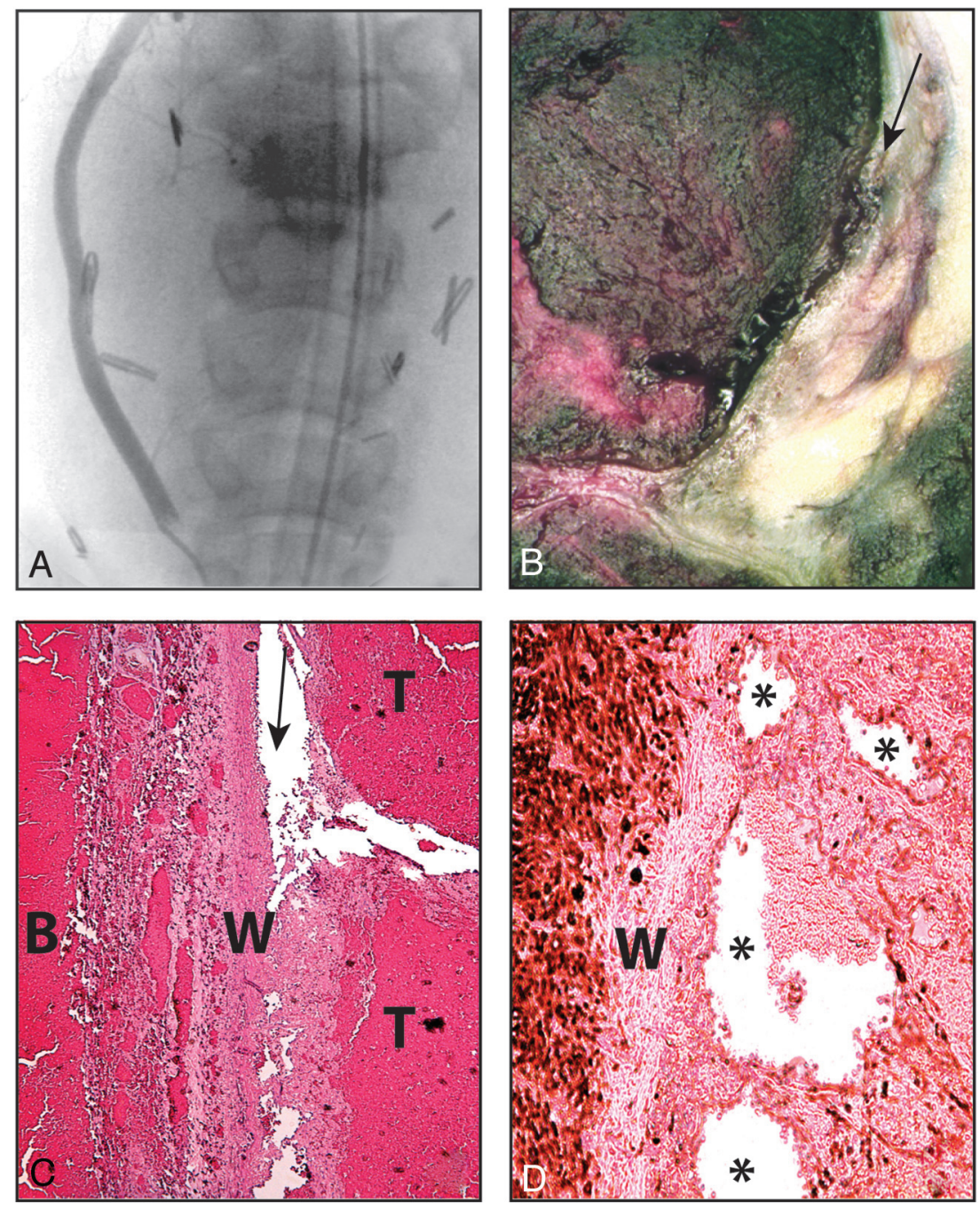

FIG 5. Bleeding despite clipping. Angiography $(A)$, sagittal section ( $B$ ), and microscopic pathology (C and $D$ ) of giant aneurysms constructed and clipped 7 days before. Note severe displacement of the right carotid artery by hematoma $(A)$, but the aneurysm is completely clipped. Recanalization between the clot and the aneurysm wall can be seen ( $B$ and $C)$. The attenuated wall (W) with intraluminal thrombosis $(T)$ and perianeurysmal blood $(B)$ is shown $(C)$. D, Microscopic sections immune-stained with $\alpha$-actin show infiltration of the clot with myofibroblasts and the formation of endothelialized vascular spaces (asterisk) between the clot and the wall (W).

early specimens difficult if not impossible; and 3) coil embolization may not fully occlude aneurysms, as previously reported. ${ }^{25}$ Other potential therapeutic avenues include endothelial denudation by using cryo- or radio-frequency ablation of the endothelial lining. ${ }^{38-39}$

\section{Limitations}

There are multiple limitations to this work, some being intrinsic to the design of the experiments. Once it was decided to compare pathologic findings on day 7 , it became impossible to learn whether unruptured aneurysms would have ruptured later. For example, denuded aneurysms could have ruptured later had they been observed for longer periods. Furthermore, fatal rupture of an aneurysm on 1 side prevented the evaluation of the natural history of the second aneurysm. Although the timing of porcine aneurysm hemorrhage was predictable, it still varied by 12-24 hours. Many ruptures occurred when an- imals were not under immediate observation. Ethical concerns regarding the circumstances surrounding animal deaths led to interruption of this exploratory research before all planned experiments could be completed. This led to disparities in group size and length of follow-up periods. This fact alone may limit the use of this model.

The present study introduces a new model that would require standardization and validation. Aneurysms are surgical constructions from vein pouches, not "natural aneurysms," and swine biology differs significantly from human biology. The evolution and pathologic results after creation of the various models are affected by multiple and complex factors, including surgical trauma, translocation of venous tissue into an arterial environment, remodelling, variable flows, partial thrombosis, and experimental artifacts; and it is impossible to disentangle confounding from true causal factors and events. Remodelling may have occurred between aneurysm construction and aneurysm rupture. This may have resulted in a change in the size and/or the geometry of the aneurysms. Reversing a venous graft, suturing venous pouches to carotid arteries, and clipping the base of the resulting lesions immediately after construction are hardly representative of clinical human aneurysms. Nevertheless, there are few if any other ways of studying ruptures in laboratory animals, and the present model and its variants could provide clues relevant to understanding rupture of human aneurysms with or without treatment.

\section{CONCLUSIONS}

Thrombosis is consistently associated with degeneration of the aneurysmal wall, but residual flows or recanalization may be required for clinical ruptures to occur.

Disclosures: Jean Raymond-UNRELATED: Grants/Grants Pending: Canadian Institutes of Health Research*, Fonds de la Recherche en Santé du Québec*, Heart and Stroke Foundation of Canada academic grant.* Tim E. Darsaut-UNRELATED: Grants/Grants Pending: project grant for Society of Interventional Radiology* Foundation/1 year/\$25,000, ended June 2011.* Guylaine Gevry—UNRELATED: Employment: Centre Hospitalier de l'Uuniversité de Montréal (CHUM) Research Centre, Comments: employee of the laboratory, paid from academic grant. *Money paid to the institution.

\section{REFERENCES}

1. Nelson PK, Lylyk P, Szikora I, et al. The Pipeline embolization device for the intracranial treatment of aneurysms trial. AJNR Am J Neuroradiol 2011;32:34-40

2. Balt Extrusion. Urgent Field Safety Notice: Intracranial stent 'SILK.' Clarifications of the indications. Letter to the intention of Hospital Chief Executives, Medical Directors and Directors of Radiology. 
Available at: http://www.mhra.gov.uk/home/groups/dts-bi/documents/ fieldsafetynotice/con076110.pdf; 2010. Accessed September 1, 2010.

3. Kulcsár Z, Houdart E, Bonafe A, et al. Intra-aneurysmal thrombosis as a possible cause of delayed aneurysm rupture after flowdiversion treatment. AJNR Am J Neuroradiol 2011;32:20-25

4. Hampton T, Walsh D, Tolias C, et al. Mural destabilization after aneurysm treatment with a flow-diverting device: a report of two cases. J Neurointerv Surg 2011;3:167-71

5. Kerber CW, Hecht ST, Knox K, et al. Flow dynamics in a fatal aneurysm of the basilar artery. AJNR Am J Neuroradiol 1996;17:1417-21

6. Vincent F, Weill A, Roy D, et al. Carotid ophthalmic aneurysm rupture after parent vessel occlusion. AJNR Am J Neuroradiol 2005;26: 1372-74

7. Ujiie $\mathrm{H}$, Sato $\mathrm{K}$, Onda $\mathrm{H}$, et al. Clinical analysis of incidentally discovered unruptured aneurysms. Stroke 1993;24:1850-56

8. Ujiie H, Tachibana H, Hiramatsu O, et al. Effects of size and shape (aspect ratio) on the hemodynamics of saccular aneurysms: a possible index for surgical treatment of intracranial aneurysms. Neurosurgery 1999;45:119-29, discussion 129-30

9. Coutard M, Touat Z, Houard X, et al. Thrombus versus wall biological activities in experimental aortic aneurysms. J Vasc Res 2010;47: $355-66$

10. Fontaine V, Jacob MP, Houard X, et al. Involvement of the mural thrombus as a site of protease release and activation in human aortic aneurysms. Am J Pathol 2002;161:1701-10

11. Lee D, Yuki I, Murayama Y, et al. Thrombus organization and healing in the swine experimental aneurysm model. Part I. A histological and molecular analysis. J Neurosurg 2007;107:94-108

12. Touat $Z$, Ollivier V, Dai J, et al. Renewal of mural thrombus releases plasma markers and is involved in aortic abdominal aneurysm evolution. Am J Pathol 2006;168:1022-30

13. Bouzeghrane F, Darsaut T, Salazkin I, et al. Matrix metalloproteinase- 9 may play a role in recanalization and recurrence after therapeutic embolization of aneurysms or arteries. J Vasc Interv Radiol 2007;18:1271-79

14. Darsaut T, Bouzeghrane F, Salazkin I, et al. The effects of stenting and endothelial denudation on aneurysm and branch occlusion in experimental aneurysm models. J Vasc Surg 2007;45:1228-35

15. Darsaut T, Salazkin I, Ogoudikpe C, et al. Effects of stenting the parent artery on aneurysm filling and gene expression of various potential factors involved in healing of experimental aneurysms. Interv Neuroradiol 2006;12:289-302

16. Darsaut TE, Bing F, Salazkin I, et al. Flow diverters can occlude aneurysms and preserve arterial branches: a new experimental model. AJNR Am J Neuroradiol 2012;33:2004-09

17. Darsaut TE, Bing F, Salazkin I, et al. Testing flow diverters in giant fusiform aneurysms: a new experimental model can show leaks responsible for failures. AJNR Am J Neuroradiol 2011;32:2175-79

18. Raymond J, Darsaut T, Salazkin I, et al. Mechanisms of occlusion and recanalization in canine carotid bifurcation aneurysms embolized with platinum coils: an alternative concept. AJNR Am J Neuroradiol 2008;29:745-52

19. Byrne JV, Hubbard N, Morris JH. Endovascular coil occlusion of experimental aneurysms: partial treatment does not prevent subsequent rupture. Neurol Res 1994;16:425-27

20. Hasan DM, Mahaney KB, Brown RD Jr, et al. Aspirin as a promising agent for decreasing incidence of cerebral aneurysm rupture. Stroke 2011;42:3156-62

21. Aoki $\mathrm{T}$, Kataoka $\mathrm{H}$, Ishibashi $\mathrm{R}$, et al. Simvastatin suppresses the progression of experimentally induced cerebral aneurysms in rats. Stroke 2008;39:1276-85
22. Tada Y, Kitazato KT, Yagi K, et al. Statins promote the growth of experimentally induced cerebral aneurysms in estrogen-deficient rats. Stroke 2011;42:2286-93

23. Raymond J, Guilbert F, Metcalfe A, et al. Role of the endothelial lining in recurrences after coil embolization: prevention of recanalization by endothelial denudation. Stroke 2004;35:1471-75

24. Raymond J, Sauvageau E, Salazkin I, et al. Role of the endothelial lining in persistence of residual lesions and growth of recurrences after endovascular treatment of experimental aneurysms. Stroke 2002;33:850-55

25. Murayama Y, Vinuela F, Suzuki Y, et al. Development of the biologically active Guglielmi detachable coil for the treatment of cerebral aneurysms. Part II. An experimental study in a swine aneurysm model. AJNR Am J Neuroradiol 1999;20:1992-99

26. Bouzeghrane F, Naggara O, Kallmes DF, et al. In vivo experimental intracranial aneurysm models: a systematic review. AJNR Am J Neuroradiol 2010;31:418-23

27. Turk AS, Aagaard-Kienitz B, Niemann D, et al. Natural history of the canine vein pouch aneurysm model. AJNR Am J Neuroradiol 2007;28:531-32

28. German WJ, Black SP. Experimental production of carotid aneurysms. $N$ Engl J Med 1954;250:104-06

29. Ryu CW, Kwon OK, Koh JS, et al. Analysis of aneurysm rupture in relation to the geometric indices: aspect ratio, volume, and volumeto-neck ratio. Neuroradiology 2011;53:883-89

30. Murayama Y, Tateshima S, Gonzalez NR, et al. Matrix and bioabsorbable polymeric coils accelerate healing of intracranial aneurysms: long-term experimental study. Stroke 2003;34:2031-37

31. Kanematsu Y, Kanematsu M, Kurihara C, et al. Critical roles of macrophages in the formation of intracranial aneurysm. Stroke 2011;42: 173-78

32. Kadirvel R, Dai D, Ding YH, et al. Endovascular treatment of aneurysms: healing mechanisms in a swine model are associated with increased expression of matrix metalloproteinases, vascular cell adhesion molecule-1, and vascular endothelial growth factor, and decreased expression of tissue inhibitors of matrix metalloproteinases. AJNR Am J Neuroradiol 2007;28:849-56

33. Cebral JR, Castro MA, Burgess JE, et al. Characterization of cerebral aneurysms for assessing risk of rupture by using patient-specific computational hemodynamics models. AJNR Am J Neuroradiol 2005;26:2550-59

34. Cebral JR, Mut F, Raschi M, et al. Aneurysm rupture following treatment with flow-diverting stents: computational hemodynamics analysis of treatment. AJNR Am J Neuroradiol 2011;32:27-33

35. Cebral JR, Mut F, Weir J, et al. Quantitative characterization of the hemodynamic environment in ruptured and unruptured brain aneurysms. AJNR Am J Neuroradiol 2011;32:145-51

36. Fiorella D, Sadasivan C, Woo HH, et al. Regarding "Aneurysm rupture following treatment with flow-diverting stents: computational hemodynamics analysis of treatment." AJNR Am J Neuroradiol 2011;32:E95-97, author reply E98-100

37. Dehdashti AR, Thines L, Willinsky RA, et al. Symptomatic enlargement of an occluded giant carotido-ophthalmic aneurysm after endovascular treatment: the vasa vasorum theory. Acta Neurochir (Wien) 2009;151:1153-58

38. Raymond J, Metcalfe A, Salazkin I, et al. Endoluminal cryotherapy to prevent recanalization after endovascular occlusion with platinum coils. J Vasc Interv Radiol 2006;17:1499-504

39. Raymond J, Savard P, Salazkin I, et al. Radiofrequency endothelial ablation prevents recanalization after endovascular coil occlusion: in vitro and in vivo assessment. J Vasc Interv Radiol 2010;21:101-77 